\title{
Estudio sistémico de la mineria aurífera artesanal y de pequeña escala en Colombia
}

\section{Systemic study of artisanal and small-scale gold mining in Colombia}

\author{
Humberto Alejandro Barrero Arciniegas ${ }^{1}$
}

(.).......

Recepción: 30/09/2020
(2)

Aprobación: 15/10/2020
(-)

Publicación: 18/12/2020

\section{Para citar este artículo:}

Barrero Arciniegas, H. A. (2020). Estudio sistémico de la minería aurífera artesanal y de pequeña escala en Colombia. Indagare, (8), 158-170. https://doi.org/10.35707/indagare/813

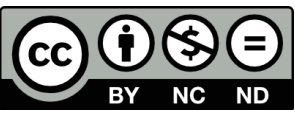

${ }^{1}$ Grupo de Investigación MYSCO, Universidad de Ibagué. ORCID: 0000-0003-3809-7998. Correo electrónico: alejandro. barrero@unibague.edu.co 
INDAGA]EE e-ISSN: 2357-5042 • Número 8 (2020) • Universidad de Ibagué • doi: https://doi.org/10.35707/indagare/813

\title{
Resumen
}

En este artículo se sintetiza un estudio sistémico que se aborda bajo la metodología propuesta por Aldana y Reyes (2004), para el planteamiento, entendimiento y comprensión de las estructuras sistémicas de la situación problemática compleja, definida como las prácticas rudimentarias en la minería aurífera artesanal y de pequeña escala con mercurio en Colombia, lugar donde la actividad ilegal e informal juegan un papel significativo.

\begin{abstract}
This article summarizes a systemicstudy that is addressed under the methodology proposed by Aldana and Reyes (2004), for the approach, understanding and comprehension of systemic structures of the complex problematic situation. It is defined as rudimentary practices in artisanal gold mining and small- scale with mercury in Colombia, a place where the illegal and informal activity play a significant role.
\end{abstract}

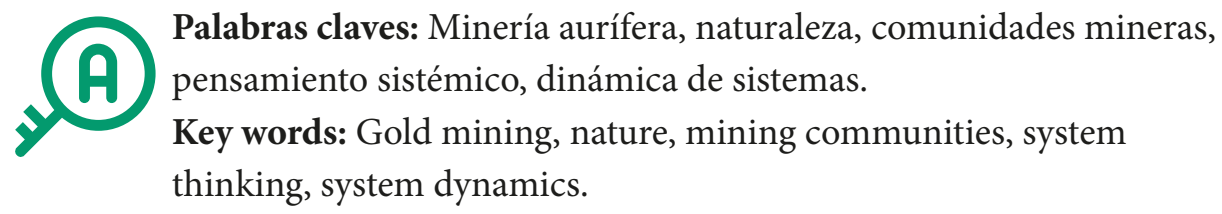

\section{Introducción}

La extracción de oro es una actividad que ha venido en aumento desde comienzos del presente siglo, tendencia que se prevé seguirá en crecimiento durante los próximos años. Una proyección de demanda de la Unidad de Planeación Minero Energética (UPME) junto a Commodities Research Unit International Limited (CRU), estima que la demanda mundial de oro pasará de 2788 toneladas en 2018 a 3373 toneladas en 2035 (CRU \& UPME, 2018). La obtención de este metal precioso se lleva a cabo, en gran parte, en países en vía de desarrollo, que, en su mayoría realizan extracción artesanal y a pequeña escala (Poveda Ávila et al., 2015). Por su parte, Colombia cuenta con ecosistemas de gran riqueza en este mineral y se posiciona como uno de los mayores productores entre los países latinoamericanos (Ministerio de Ambiente y Desarrollo Sostenible, 2012).

Una revisión de la literatura indica que distintos trabajos han centrado esfuerzos en la descripción y caracterización de la realidad que se vive en el sector mineroaurífero a nivel artesanal y de pequeña escala. En estos se han constatado las prácticas rudimentarias 
que se efectúan para la extracción del oro y sus efectos negativos tanto para el medio ambiente como para la salud humana. A la vez, se presentan alteraciones en el orden social, puesto que, la mayoría de estas labores son realizadas de forma ilegal e informal, bajo la coordinación de grupos al margen de la ley, para quienes el mercurio se presenta como una sustancia accesible y muy efectiva en el desarrollo de esta labor extractivista (Ministerio de Ambiente y Desarrollo Sostenible, 2012; Programa de las Naciones Unidas para el Medio Ambiente, 2008).

Las zonas más afectadas por estas problemáticas se encuentran en los departamentos del Chocó, Valle del Cauca y Nariño; sin embargo, territorios con gran producción de oro como Antioquia, Bolívar, Caldas, Cauca, Tolima, Córdoba, Santander, Risaralda, Putumayo y Huila, no están del todo exentos y, por consecuente, también se contemplan en esta investigación (Ministerio de Ambiente y Desarrollo Sostenible, 2012).

En lo referente a estudios sistémicos sobre la minería aurífera, se evidenció una propuesta para el análisis de la productividad de un proceso mineroaurífero colombiano, por medio del cual se realiza la caracterización del sistema y la evaluación de políticas para su mejoramiento (Delgado Álvarez, Arango Aramburo \& Romero Hernández, 2014). En términos generales, la revisión de la literatura indica la ausencia de artículos, informes y publicaciones que consideran las interrelaciones sistémicas en el ámbito social, económico, político, cultural, ambiental y sanitario, que se han formado respecto a la producción y comercialización del oro. Tampoco se evidencian investigaciones orientadas a comprender la estructura del sistema que engloba la minería aurífera artesanal y de pequeña escala en Colombia.

El presente trabajo, bajo la pregunta ¿qué estructuras sistémicas conforman las prácticas rudimentarias en la minería aurífera artesanal y de pequeña escala con mercurio en Colombia?, es un esfuerzo por aportar a la literatura en aras de comprender la estructura del sistema alrededor de este tipo de minería realizada desde esas prácticas. Así, se facilita su entendimiento y aproximación desde su estructura, para contar con la posibilidad de transformar el pensamiento frente a este tipo de situaciones problemáticas complejas con miras a la construcción de un buen juicio.

\section{Materiales y métodos}

Este es un estudio que se aborda bajo la metodología propuesta por Aldana y Reyes (2004), que sugiere un conjunto de herramientas que permiten guiar la comprensión de situaciones problemáticas complejas bajo un enfoque sistémico. La presente situación se caracteriza por ser transdisciplinaria, lo cual devela la complejidad allí inmersa e implica 
INDAGAZEE e-ISSN: 2357-5042 • Número 8 (2020) • Universidad de Ibagué • doi: https://doi.org/10.35707/indagare/813

que su descripción dependa, no solo del punto de vista en que se observe, sino también de las interacciones entre las personas involucradas. Dichas personas son en su mayoría actores sociales relevantes ${ }^{2}$ que se relacionan de forma recurrente, lo cual conlleva a que fruto de estas interrelaciones emerja la problemática. Esto es muy importante, pues de acuerdo con los autores, comprender una situación de este tipo implica conocer cuáles son sus actores sociales relevantes, hacer explícitas las relaciones que sostienen a través del tiempo y sintetizar sus descripciones de la situación problemática, es decir, se requiere de una observación sistémica más que de una observación analítica (Aldana \& Reyes, 2004).

Por ende, en el presente estudio sistémico es relevante tener en cuenta las diferentes perspectivas alrededor de la problemática. Se lleva a cabo tanto la identificación de los actores sociales relevantes, como de las variables relevantes ${ }^{3}$ y sus relaciones. En adición, se clarifican las tres cadenas lógicas - manifestaciones, causas, consecuencias- y del conjunto de herramientas propuestas en dicha metodología para describir la situación problemática, se efectúa la aplicación del diagrama causa-efecto y el diagrama de ciclos causales. A través de este último, se ilustra un modelo que intenta representar la realidad que se evidencia en dicha problemática. Esta herramienta es netamente cualitativa y es de uso generalizado del lenguaje de dinámica de sistemas creado por Jay W. Forrester (Forrester, 1961) y popularizada por Peter Senge, en su obra The Fifth Discipline: The Art \& Practice of the Learning Organization (Senge, 1990).

\section{Resultados}

\subsection{Actores sociales relevantes de la problemática en estudio}

Las prácticas rudimentarias en la minería aurífera artesanal y de pequeña escala con mercurio en Colombia están, en esencia, enraizadas en las relaciones que sostienen los actores involucrados en esta actividad extractivista. Por ende, siguiendo la metodología propuesta por Aldana y Reyes, se implementó la heurística sugerida para el proceso de determinación de actores y variables relevantes, que consiste en responder de forma cuidadosa un conjunto de preguntas (Aldana \& Reyes, 2004).

\footnotetext{
${ }^{1}$ Actores relevantes: son las personas que pueden afectar o ver afectadas por la situación problemática (Aldana \& Reyes, 2004).

${ }^{2}$ Variables relevantes: conjunto de aspectos medibles - cuantitativa o cualitativamente- que permiten apreciar cambios en la situación problemática en el tiempo (Aldana \& Reyes, 2004).
} 
Gracias a lo anterior, se identificaron como actores relevantes las comunidades allegadas a los territorios en donde se realiza la explotación minera de oro con mercurio y que no participan de ella, porque se ven perjudicadas con esa situación en los ámbitos social, ambiental y de salud humana, al igual que las comunidades mineras. Sin embargo, estos últimos también se ven beneficiados debido a los ingresos monetarios que perciben al ejercer la labor extractivista, igual sucede para el caso de los grupos al margen de la ley. Sumado a esto, el Gobierno colombiano, junto con el Ministerio de Minas y Energía, el Ministerio de Ambiente y Desarrollo Sostenible, la Policía Nacional y el Ejército Nacional, resultan ser actores relevantes, porque son quienes pueden determinar y modificar su contexto normativo. Es de aclarar que todos ellos pueden asignar, distribuir o regular recursos que sostienen dicha situación en el tiempo.

\subsection{Manifestaciones, causas y consecuencias de la problemática en estudio}

Algunas de las manifestaciones de la problemática en estudio son las grandes cantidades de mercurio que se utilizan en este tipo de minería (artesanal y de pequeña escala) en gran parte del territorio colombiano. De acuerdo con Mercury Watch, en Colombia hubo un consumo promedio de 180 toneladas de mercurio en 2013, se estima que se consumen entre tres y cuatro toneladas de mercurio por cada tonelada de oro extraído (Poveda Ávila et al., 2015). También, se evidencia la implementación de maquinaria como dragas, retroexcavadoras, motobombas y volquetas controladas en muchos casos por grupos armados ilegales articulados a redes internacionales de lavado del narcotráfico (Semana Sostenible, 2019).

En suma, se presenta el hecho de que los mineros no cuentan con las condiciones de salubridad laborales que exige la ley. Si se tiene en cuenta que las condiciones laborales y de seguridad industrial son inadecuadas, quedan expuestos a graves efectos perjudiciales para su salud. Por otra parte, se refleja la destrucción y degradación de los ecosistemas, acciones que permiten evidenciar una considerable afectación en la geografía de los territorios donde se lleva a cabo la extracción del oro (Ministerio de Ambiente y Desarrollo Sostenible, 2012).

Después, a través de un diagrama causa-efecto, se pretende continuar describiendo la situación problemática en estudio, la cual se puede observar en las circunferencias de las figuras 1 y 2 . Las causas y subcausas se observan en la Figura 1. Las consecuencias junto a sus subconsecuencias se ilustran en la Figura 2. La primera causa es el aumento de la producción de oro, entre sus subcausas se encuentra que se debe a su gran demanda tecnológica, puesto que este metal precioso se emplea en la fabricación de equipos electrónicos gracias a que sus propiedades de resistencia a la corrosión, alta conductividad 
INDAGA]EE e-ISSN: 2357-5042 • Número 8 (2020) • Universidad de Ibagué • doi: https://doi.org/10.35707/indagare/813

eléctrica y buen grado de maleabilidad, hacen de él el material por excelencia para la fabricación de microcables de los microcircuitos electrónicos o microchips (Poveda Ávila et al., 2015). Otra subcausa se debe a que los bancos centrales nacionales mantienen grandes reservas de oro, porque es el valor refugio por seguridad tanto de inversores como de los países frente a posibles crisis económicas (Poveda Ávila et al., 2015).

También, el oro destinado para la joyería representa una porción importante, porque su porcentaje de demanda mundial, que corresponde a la producción de joyas entre 2004-2013, supera el $70 \%$ en todos los años (Poveda Ávila et al., 2015). Por lo tanto, debido a las tres subcausas expuestas, y teniendo en cuenta que esta es una sociedad que le da un alto valor a este metal precioso, es que se ha presentado un aumento en su producción. Además, que ha venido en alza su precio y, en ese sentido, se presenta un escenario para una mayor inversión en su extracción. Es de destacar que Colombia pasó de producir 23,6 toneladas de oro en 2004, a producir 40,4 toneladas en 2013, cantidad que lo ubicó como el quinto mayor productor a nivel de Sudamérica (Poveda Ávila et al., 2015).

Figura 1. Causas y subcausas de la situación problemática en estudio
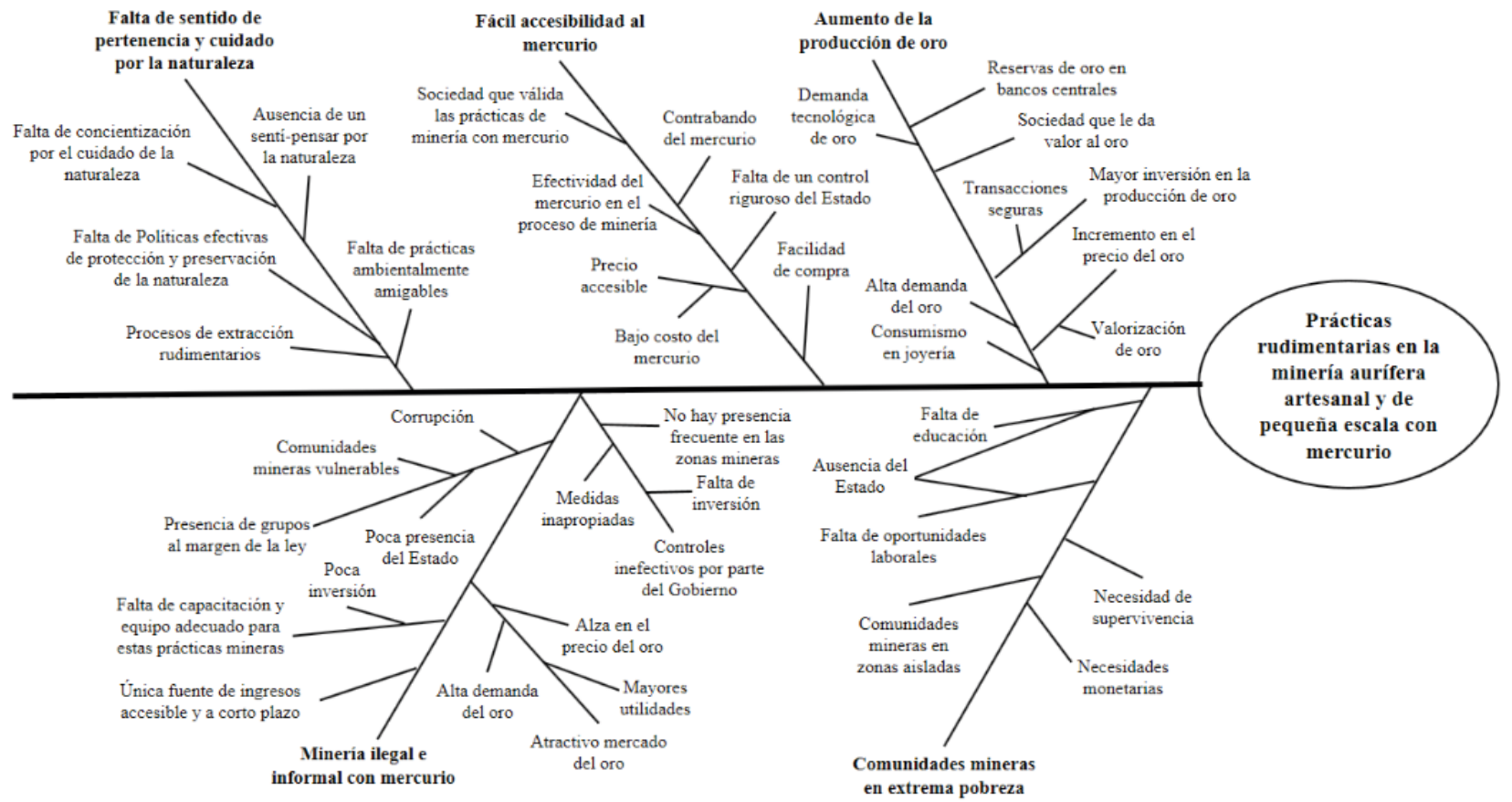

Fuente: elaboración propia 
INDAGA\}E e-ISSN: 2357-5042 • Número 8 (2020) • Universidad de Ibagué • doi: https://doi.org/10.35707/indagare/813

La gravedad de la crisis socioeconómica en las comunidades mineras en extrema pobreza es otra causa que influye de forma considerable en las labores mineroauríferas artesanales y de pequeña escala con mercurio. De acuerdo con lo anterior, este tipo de minería es una fuente de ingresos importante para esas comunidades, puesto que es su principal actividad económica para subsistir (Ministerio de Ambiente y Desarrollo Sostenible, 2012).

La minería ilegal e informal con mercurio es otra causa crítica porque más allá de generar considerables impactos sociales, ambientales y de salud pública, hay poca o nula presencia del Estado para el control de este tipo de minería en los territorios mineros. Estas zonas en su mayoría son controladas por grupos al margen de la ley con el objetivo de tener una fuente de ingresos de fácil acceso y a corto plazo, gracias a la extracción de oro de forma rudimentaria (Poveda Ávila et al., 2015).

Frente a este panorama, las intervenciones del Estado no solo han sido inefectivas sino inapropiadas, puesto que, con el apoyo de la Policía Nacional y las Fuerzas Militares, se ha destruido la maquinaria que estos grupos al margen de la ley y los mineros informales utilizan para su labor extractivista del oro. De este modo, se ha llevado a cabo la destrucción o incautación de dragas, excavadoras, motores y maquinaria utilizada para dicha extracción ilegal (Semana Sostenible, 2019)

Además, existe una fácil accesibilidad por parte de los grupos al margen de la ley en la adquisición del mercurio, que por lo regular es utilizado en grandes cantidades para procesar el mineral, a menudo en condiciones de gran inseguridad y peligrosas para el medio ambiente. Desafortunadamente el uso de mercurio es en general, el método dominante y preferido de extracción de oro en el sector de la minería artesanal y en pequeña escala, y se considera fácil de utilizar y no es costoso (Programa de las Naciones Unidas para el Medio Ambiente, 2008). Luego, como se puede evidenciar en la Figura 1, la última causa se presenta como la falta de sentido de pertenencia y cuidado por la naturaleza de parte de los seres humanos, quienes por lo general ven el medio ambiente como una cosa aparte de ellos, de la que pueden disponer a su antojo para favorecer intereses particulares (Angarita Ibarbuen, et al., 2019).

Se entiende entonces que las cinco causas expuestas con antelación junto con sus diferentes subcausas son el porqué de la situación problemática en estudio, es decir, se describieron con profundidad estos conjuntos de problemas que, como dirían Aldana y Reyes (2004), anteceden en términos temporales a la situación analizada y su presencia aumenta la probabilidad de que este ocurra.

Respecto a las consecuencias, la primera de ellas es el impacto sobre el medio ambiente, dado que, este es el entorno donde principalmente se libera el mercurio en grandes cantidades, contaminando el agua y los suelos. Esto se debe a que en el proceso 
INDAGA]E e-ISSN: 2357-5042 • Número 8 (2020) • Universidad de Ibagué • doi: https://doi.org/10.35707/indagare/813

de amalgamación el metal líquido se utiliza para adherirse al oro y poder extraerlo de la roca, sin embargo, solo el $10 \%$ del mercurio queda en la amalgama, el $90 \%$ restante se libera al ambiente. Acto seguido, esta amalgama de oro y mercurio se somete a altas temperatura al aire libre, lo cual libera al ambiente el mercurio evaporado y así obtener el metal precioso (Programa de las Naciones Unidas para el Medio Ambiente, 2008).

Figura 2. Consecuencias y subconsecuencias de la situación problemática en estudio

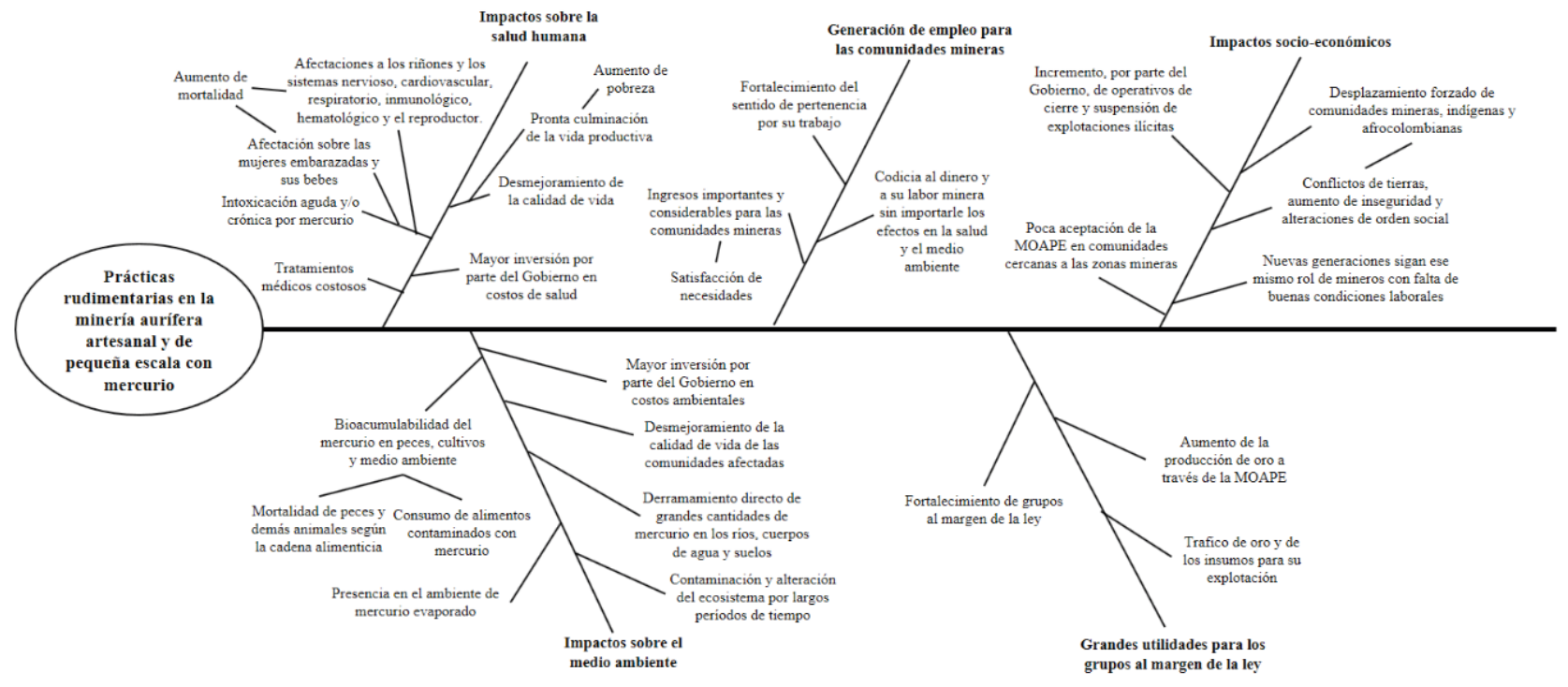

Fuente: elaboración propia

Con claridad este proceso contamina y altera el ecosistema por largos periodos, razón por la cual se presenta la dispersión del mercurio en los sistemas acuáticos, que contribuyen a la contaminación por metilmercurio de los peces, la fauna y flora silvestres. En paralelo, estos altos niveles de mercurio que se han propagado en el medio ambiente, generan un grave impacto sobre la salud humana, en especial, en las comunidades mineras y poblaciones aledañas (Olivero Verbel \& Johnson Restrepo, 2002).

En muchas comunidades se destacan los considerables ingresos económicos que tienen las familias mineras al desempeñarse en la labor extractivista, puesto que podrán satisfacer sus necesidades (Poveda Ávila et al., 2015). Esto a la vez provoca que, al percibir una estabilidad económica, se enfoquen solo en el dinero sin importarle los efectos negativos sobre su salud o el medio ambiente, los cuales muchas veces desconocen. De igual manera, gracias a la minería ilegal e informal llevada a cabo bajo el mando 
de grupos al margen de la ley, estos obtienen cuantiosas utilidades monetarias en el mercado del oro, acción que los fortalece como grupo ilegal en términos económicos y de poder, además de que podrán realizar mayores inversiones en material e insumos para su explotación y de esta manera producir más toneladas del metal precioso.

Para finalizar, como consecuencia también se presenta un impacto negativo a nivel social en los territorios mineros, puesto que en muchos casos los grupos al margen de la ley proceden a desplazar de una manera forzada a las comunidades mineras, indígenas y afrocolombianas para ocupar sus territorios y poder efectuar su labor extractivista del oro o, en algunas ocasiones, solo los someten a trabajar para ellos. Esto conlleva que se presenten conflictos de tierras, aumento de inseguridad y alteraciones de orden social en dichas regiones mineras (Defensoría del Pueblo, 2015).

\subsection{Modelo del sistema dinámico complejo de la problemática en estudio}

El diagrama de ciclos causales ilustrado en la Figura 3 permite representar relaciones más elaboradas entre variables pertinentes de la situación problemática en estudio. Este tipo de relaciones son llamadas circulares, en razón de que una variable afecta de forma causal a otra y esta acción se repite de manera sucesiva, hasta que la variable al final de la cadena afecta a la primera de ellas y forma un circuito (Aldana \& Reyes, 2004).

El diagrama de ciclos causales ilustrado en la Figura 3 presenta un modelo de la dinámica en la que se encuentra el sistema que engloba las prácticas rudimentarias de la minería de oro artesanal y de pequeña escala con mercurio en Colombia en la actualidad, por lo tanto, este es un planteamiento de la hipótesis dinámica ${ }^{4}$ que explica el comportamiento actual de esa estructura sistémica, que también se puede evidenciar de forma dinámica en el modelo realizado en Loopy (clic aquí).

B1 es un ciclo de realimentación de balance para la estabilidad económica de las comunidades mineras (nivel de pobreza de las comunidades mineras, grado de necesidad de supervivencia en las comunidades mineras, porcentaje de minería ilegal e informal, utilidades). Evidencia cómo estas comunidades a través de su labor mineroaurífera artesanal y de pequeña escala de forma ilegal e informal perciben ingresos monetarios gracias a las utilidades de esta actividad económica y así pueden disminuir su nivel de pobreza.

\footnotetext{
${ }^{1}$ Una hipótesis dinámica es una explicación provisional (sujeta a revisión o abandono) de la dinámica que caracteriza la problemática en términos de la retroalimentación subyacente en la estructura del sistema (Sterman, 2000).
} 
INDAGA]E e-ISSN: 2357-5042 • Número 8 (2020) • Universidad de Ibagué • doi: https://doi.org/10.35707/indagare/813

Figura 3. Diagrama de ciclos causales de la situación problemática en estudio

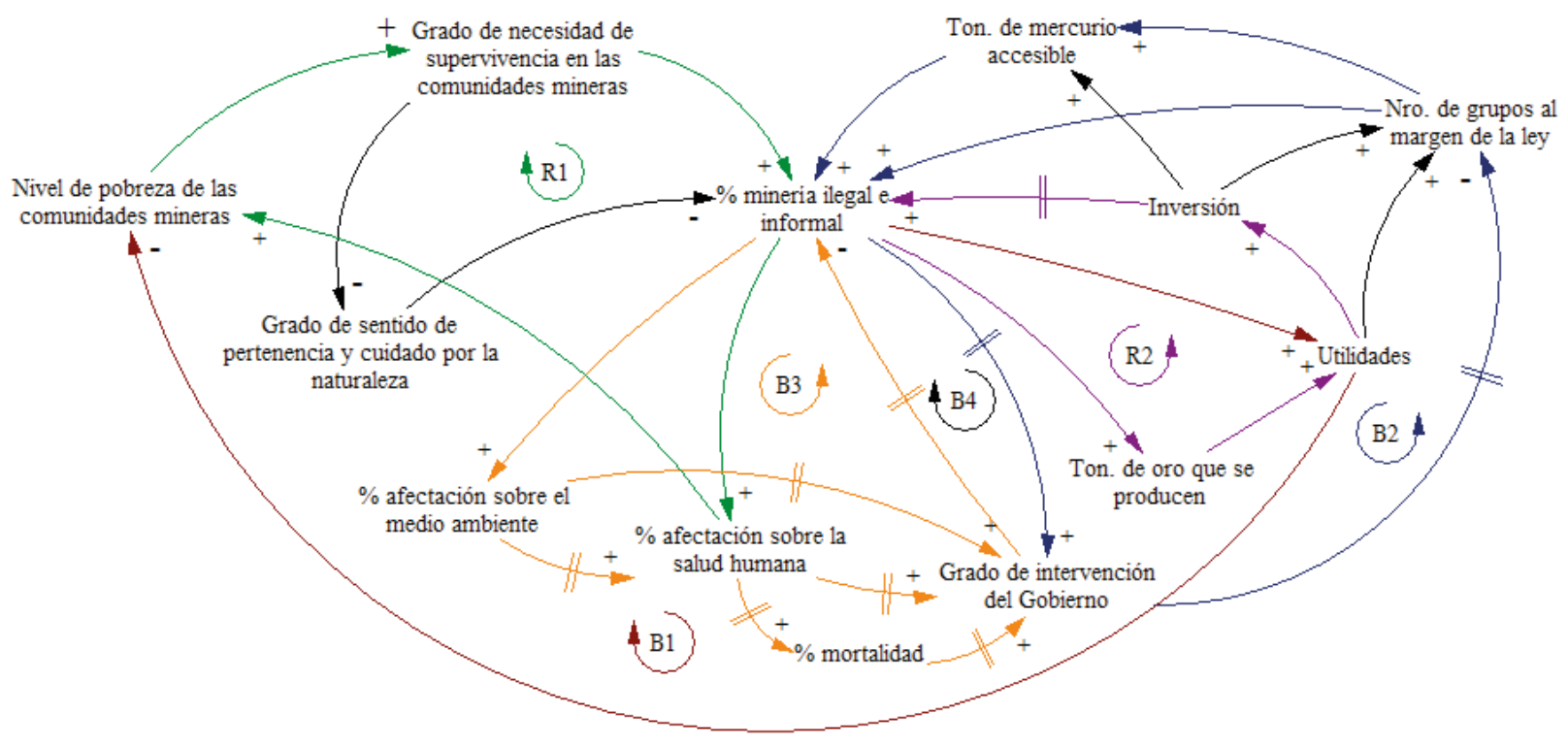

Fuente: elaboración propia

Sin embargo, el ciclo de realimentación de refuerzo R1 interpela a B1, debido a que este describe cómo puede repercutir la afectación en la salud humana de las comunidades mineras en su nivel de pobreza. Los mineros y sus familias, al tener una perjudicial exposición al mercurio, incurren en graves enfermedades que acarrean considerables gastos de salud, o en su defecto, no podrán continuar con esta labor extractivista y, por ende, dejarán de percibir ingresos que los llevará a tener un mayor nivel de pobreza.

Por su parte, el ciclo B2 presenta la reducción en el número de grupos al margen de la ley, gracias a la intervención del Gobierno colombiano a través de sus operativos articulados con la Policía y el Ejército Nacional, lo cual se lleva a cabo con ciertas demoras tanto en enterarse de las zonas donde se realiza la minería ilegal e informal, así como de la ejecución de esta intervención que conllevará la disminución del número de grupos al margen de la ley, de forma parcial.

No obstante, la dinámica de B2 no es favorecida por R2, puesto que este ciclo evidencia cómo, gracias a las utilidades generadas por la producción de oro, se tiende a aumentar el número de grupos al margen de la ley que promuevan esta actividad minera, dado que es un negocio muy atractivo por su capacidad lucrativa. Además, habrá una mayor cantidad de dinero disponible para una mayor inversión, con la cual, después de un tiempo mejorará y aumentará el porcentaje de minería ilegal e informal. 
B3 es el ciclo de la afectación al medio ambiente y la salud humana por la minería de oro ilegal e informal con mercurio. Este es de balance, porque pasado un tiempo de demora, en el cual el Gobierno se percate de esta afectación, aumentará su grado de intervención para disminuir el porcentaje de este tipo de minería, lo cual conlleva disminuir la afectación mencionada. Sin embargo, se debe tener en cuenta que hay un tiempo que se invierte en la identificación de los territorios perjudicados, así como la organización y ejecución de los operativos correspondientes por parte del Estado.

Diagramas de este tipo son muy útiles para seguir enriqueciendo nuestra comprensión de la situación problemática, en este caso específico, observando la dinámica del sistema que se gesta alrededor de la minería de oro artesanal y de pequeña escala con mercurio en Colombia. Por lo tanto, resulta valioso el análisis particular de las relaciones entre las variables relevantes de dicha problemática y cómo determinan el comportamiento del sistema en estudio.

La variable de la minería aurífera artesanal y de pequeña escala basada en prácticas rudimentarias con el uso de mercurio llevada a cabo, en gran parte de forma ilegal e informal, tiende a incrementar influenciada con frecuencia por un alto nivel de pobreza en las comunidades mineras. A la vez, una cantidad considerable de grupos al margen de la ley que promueven esta actividad minera, de la que obtienen cuantiosas utilidades que conllevan reforzar el ciclo de realimentación que favorece el incremento de este tipo de minería, sobre la cual el Gobierno colombiano interviene de una forma tardía y poco efectiva.

Por último, la complejidad evidenciada dada la interconexión de estos sistemas de problemas también nos lleva a concluir que la problemática alrededor de las prácticas rudimentarias en la minería aurífera artesanal y de pequeña escala con mercurio en Colombia es una manifestación de un problema más de fondo, que se podría describir como la pérdida de sentido de pertenencia y cuidado de la naturaleza por parte del ser humano. Esto obedece a diferentes dinámicas socioeconómicas en las que los hombres y las mujeres han estado inmersos, las cuales los ha promovido a ver la naturaleza como un simple recurso que puede explotar para su beneficio monetario, o en su defecto, para satisfacer gustos innecesarios en la mayoría de los casos.

\section{Potencial uso}

La aplicación de la metodología propuesta por Aldana y Reyes (2004) permite alcanzar un sensato nivel de estabilidad en la comprensión de la problemática que concierne a las prácticas rudimentarias en la minería aurífera artesanal y de pequeña escala con mercurio en Colombia. De ella resulta trascendental el uso de las herramientas más adecuadas para 
INDAGAZEE e-ISSN: 2357-5042 • Número 8 (2020) • Universidad de Ibagué • doi: https://doi.org/10.35707/indagare/813

reconocer las diversas perspectivas de los actores sociales relevantes involucrados, puesto que es fundamental la comprensión de su estructura sistémica y facilita la posibilidad de transformar el pensamiento frente a este tipo de situaciones problemáticas complejas. Esto se lleva a cabo con miras a la construcción de un buen juicio que favorezca una adecuada intervención en pro de una equidad social, que reconoce las realidades de los involucrados y promueve una transformación social que coconstruye comunidades autónomas.

\section{Agradecimientos}

El autor de este trabajo agradece de forma especial el apoyo y acompañamiento del doctor Hernán López-Garay; a los estudiantes del programa de Diseño: Jesús David Angarita Ibarbuen, Laura Natalia Charry Rico, Camila Fernández Zarta, Juan Sebastián Gutiérrez Peñaloza, Vanessa Morán Fayad, Sebastián Ramos Reyes; a la profesora María Juliana Rojas Salgado y a mi gran amiga Liliana Paola Prada Penagos.

\section{Referencias}

Aldana, E., \& Reyes, A. (2004). Disolver problemas: criterio para formular proyectos sociales. Recuperado de https://bit.ly/3kBRpBS

Angarita Ibarbuen, J. D., Charry Rico, L. N., Fernández Zarta, C., Gutiérrez Peñaloza, J. S., Morán Fayad, V., \& Ramos Reyes, S. (2019). Magua. De moléculas a transformación social. Recuperado de https://n9.cl/7nlqb

CRU \& UPME. (2018). Oro. Caracterización y análisis de mercado internacional de minerales en el corto, mediano, y largo plazo con vigencia al año 2035. Recuperado de https://bit.ly/2HIBkeW

Defensoría del Pueblo. (2015). La minería sin control. Un enfoque desde la vulneración de los derechos humanos. Bogotá, Colombia: Imprenta Nacional de Colombia.

Delgado-Álvarez, C. A., Arango-Aramburo, S., \& Romero-Hernández, A. (2014). Una propuesta sistémica para el análisis de la productividad de un proceso minero aurífero colombiano. Revista Facultad de Ingeniería Universidad de Antioquia, (72), 173-185. Recuperado de https://revistas.udea.edu.co/ index.php/ingenieria/article/view/16711

Forrester, J. (1961). Industrial Dynamics. Cambridge, USA: The MIT Press.

Ministerio de Ambiente y Desarrollo Sostenible. (2012). Sinopsis nacional de la minería aurífera artesanal $y$ de pequeña escala. Recuperado de https://n9.cl/9dwy7

Olivero Verbel, J., \& Johnson Restrepo, B. (2002). El lado gris de la minería del oro: la contaminación con mercurio en el norte de Colombia. Cartagena, Colombia: Editorial Universitaria.

Poveda Ávila, P., Córdova Eguivar, H., Pulido, A., Sacher, W., Oliveira Neves, L. J., Daró, E., \& Marchegiani, P. (2015). La economía del oro: ensayos sobre la explotación en Sudamérica. La Paz, Bolivia: Editorial CEDLA. 
Programa de las Naciones Unidas para el Medio Ambiente. (2008). El uso del mercurio en la minería del oro artesanal y en pequeña escala. Módulo 3. Recuperado de https://bit.ly/3gVjnHN

Semana Sostenible. (21 de marzo de 2019). Luchan contra la minería ilegal en Chocó. Semana Sostenible. Recuperado de https://n9.cl/2hi9

Senge, P. (1990). The Fifth Discipline: The Art \& Practice of the Learning Organization. New York, USA: Doubleday Dell Publishing Group.

Sterman, J. (2000). Business Dynamics: Systems Thinking and Modeling for a Complex World. New York, USA: Irwin/McGraw-Hill. 